\title{
Osteocalcin Induces Proliferation via
} Positive Activation of the PI3K/Akt, P38 MAPK Pathways and Promotes Differentiation Through Activation of the GPRC6A-ERK1/2 Pathway in C2C12 Myoblast Cells

\author{
Suifeng Liua,b Feng Gao ${ }^{\mathrm{a}}$ Lei Wen ${ }^{\mathrm{c}}$ Min Ouyang ${ }^{\mathrm{a}} \quad$ Yi Wang $^{\mathrm{a}}$ Qiong Wang \\ Liping Luo ${ }^{d}$ Zaijin Jian ${ }^{a}$ \\ aDepartment of Geriatrics, The Second Xiangya Hospital, Central South University, Changsha, \\ ${ }^{b}$ Department of Cardiology, Zhongshan Hospital, Xiamen University, Xiamen, 'Medical College of Xiamen \\ University, Xiamen, 'Metabolic Syndrome Research Center of Central South University, Changsha, China
}

\section{Key Words}

Osteocalcin - Myoblast - Proliferation - Differentiation - GPRC6A - PI3K/Akt - P38 MAPK • ERK1/2

\begin{abstract}
Background/Aims: Sarcopenia is characterized by an age-related decline in skeletal muscle plus low muscle strength and/or physical performance. Despite the clinical significance of sarcopenia, the molecular pathways underlying sarcopenia remain elusive. The recent demonstration that undercarboxylated osteocalcin (ucOC) favours muscle function related to insulin sensitivity and glucose metabolism raises the question of whether this hormone may also regulate muscle mass. The present study explored the promotive effects of ucOC in proliferation and differentiation processes of $\mathrm{C} 2 \mathrm{C} 12$ myoblasts as well as the possible signalling pathways involved. Methods: The effects of exogenous ucOC on $\mathrm{C} 2 \mathrm{C} 12$ myoblasts proliferation were assessed using CCK8 and immunohistological staining assays. C2C12 cells were pretreated with PI3K/Akt or P38 MAPK inhibitors to investigate the possible involvement of the PI3K/Akt and P38 MAPK pathways in proliferation. The levels of Akt, phosphorylatedAkt (p-Akt), P38, and phosphorylated-P38 (p-P38) were measured by Western Blotting. The effects of ucOC on myoblast differentiation were quantified by morphological analysis. $A$ silencing experiment was conducted in which the expression of GPRC6A in C2C12 myoblasts was modified. The expression of GPRC6A, myosin heavy chain (MyHC) and the related ERK1/2 signalling pathway in C2C12 myoblasts were monitored by qRT-PCR and Western Blotting. Results: We showed that treatment with exogenous ucOC stimulated the priming

Zaijin Jian,

Department of Geriatrics, The Second Xiangya Hospital, Central South University, 139 Middle Renmin Road, Changsha, (China)

Tel. +86 13950131145, Fax +86-592-2590228, E-Mail liusuisui@yahoo.com
\end{abstract}

KARGER 
of $\mathrm{C} 2 \mathrm{C} 12$ myoblasts proliferation. Inhibition of Akt phosphorylation by wortmannin or inhibition of P38 MAPK phosphorylation by SB203580 decreased C2C12 cell proliferation. Wortmannin also reduced P38 MAPK phosphorylation, whereas SB203580 did not affect Akt activation. Furthermore, uCOC promoted $\mathrm{C} 2 \mathrm{C} 12$ myoblast differentiation. Inhibition of ERK1/2 phosphorylation with U0126 decreased C2C12 cell differentiation. Finally, GPRC6A expression was substantially increased after ucOC treatment of $\mathrm{C} 2 \mathrm{C} 12$ cells. GPRC6A silencing inhibited Akt, P38 MAPK phosphorylation in $\mathrm{C} 2 \mathrm{C} 12$ cells, and ERK1/2 phosphorylation in $\mathrm{C} 2 \mathrm{C} 12$ myotubes; GPRC6A silencing also decreased cell proliferation, decreased cell differentiation, and downregulated $\mathrm{MyHC}$ expression. Conclusions: The present data suggest that ucOC induces myoblast proliferation via sequential activation of the PI3K/Akt and p38 MAPK pathways in C2C12 myoblast cells. Moreover, ucOC enhances myogenic differentiation via a mechanism involving GPRC6A-ERK1/2 signalling.

\section{Introduction}

During ageing, a progressive loss of skeletal muscle mass and a parallel decrease in muscle strength and endurance occur [1]. This condition, termed sarcopenia, has important healthcare and socioeconomic implications for humans as it contributes to frailty, functional loss, disability, high healthcare costs, and premature death [2-4]. Despite the clinical significance of sarcopenia, the mechanisms through which sarcopenia develops are complex and still remain to be completely elucidated, and standardized intervention programmes are still lacking. Bone and muscle are neighbours with close ties. We and others have shown that decreases in muscle and bone mass accompany ageing [5-7]. Given the scope of the problem, it is critical to obtain a better understanding of how these two tissues integrate and crosstalk as a basis for identifying new therapeutics that can be used to prevent the loss of muscle and bone with ageing.

We already know that the interactions between muscle and bone are mediated by a variety of hormones, cytokines and nutrients [8]. However, the precise mechanisms responsible for synchronizing bone and skeletal muscle mass remain unclear. Although basic studies have helped dissect the multiple influences of skeletal muscle on bone through cytokines such as myostatin, insulin-like growth factor 1 (IGF-1), fibroblast growth factor 2 (FGF-2), interleukin-6 (IL-6), IL-15, myostatin, osteoglycin (OGN), FAM5C, and osteoactivin [9-14], few osteokines involved in the feedback control of muscle by bone have been identified, with the exception of the osteocyte-specific Wnt family member 3a (Wnt3a), prostaglandin E2 (PGE2), osteoblast-derived IGF-1 and osteocalcin [15-18].

Osteocalcin is a bone matrix protein that was initially believed to be a measure of bone mineralization [19]. Recent increasing data have emerged that cast osteocalcin in a new light; these data suggest a broader role for osteocalcin that extends to the regulation of energy metabolism, reproduction, and cognition [20-23]. An undercarboxylated form of osteocalcin (ucOC), which is the hormonally active isoform, acts in a feed-forward loop to increase $\beta$-cell proliferation as well as insulin production and secretion, and skeletal muscle and adipose tissue respond to ucOC by increasing their sensitivity to insulin [24]. More recently, an additional role for ucOC in maintaining muscle mass and function in older mice has been suggested because mice lacking osteocalcin (Ocn-/-) display decreased muscle mass and strength compared to their WT littermates [25]. Moreover, mice lacking a neuromuscular gap junction protein (connexin43) had increased muscle area and grip strength after injection with human ucOC compared to the same knockout mice injected with saline [26]. It is known that muscle mass is associated with the number and/or differentiation efficiency of myoblasts, which are the main contributors to myogenesis. However, the precise role of osteocalcin in the biological processes that occur in muscle remains to be deciphered.

The GPCR class C group 6 subtype A (GPRC6A) protein is a member of a family of G protein-coupled receptors that are sensitive to pertussis toxin [27]. GPRC6A, as the putative receptor for osteocalcin, is expressed by a wide variety of cell types, including pancreas, 
Liu et al.: Osteocalcin Induces Proliferation and Promotes Differentiation in C2C12 Myoblast Cells

testes and other tissues that respond to circulating osteocalcin $[28,29]$. It appears likely that GPRC6A also mediates osteocalcin's functions in muscle [30]. For the first time, we investigated the role of ucOC in myoblast proliferation and differentiation using $\mathrm{C} 2 \mathrm{C} 12$ cells, a recognized in vitro model of skeletal muscle. Here, we show that exogenous ucOC is sufficient to induce proliferation in $\mathrm{C} 2 \mathrm{C} 12$ cells. Furthermore, during the differentiation stage, osteocalcin promotes myogenic differentiation. These results expand the importance of the regulation of muscle physiology by bone-derived hormones and suggest a basis for novel and adapted therapies to treat age-related muscle wasting.

\section{Materials and Methods}

\section{Cell culture}

The murine myoblast cell line C2C12 was obtained from the American Type Culture Collection (Manassas, VA, USA). C2C12 cells were cultured in 100-mm culture dishes in growth medium (GM) consisting of Dulbecco's modified Eagle's medium (DMEM, HyClone, Logan, UT, USA) with 10\% foetal bovine serum (GIBCO, Grand Island, NY, USA), $100 \mathrm{U} / \mathrm{mL}$ penicillin and $100 \mu \mathrm{g} / \mathrm{mL}$ streptomycin (Solarbio, Beijing, China) under a humidified atmosphere of $5 \% \mathrm{CO} 2$ and $95 \%$ air at $37^{\circ} \mathrm{C}$. The medium was replaced every 72 hours. To induce differentiation, the cells were grown to $75 \%$ confluence, and the medium was then replaced with differentiation medium (DM) consisting of DMEM containing $\%$ horse serum (Invitrogen, Carlsbad, CA, USA), $100 \mathrm{U} / \mathrm{mL}$ penicillin and $100 \mu \mathrm{g} / \mathrm{mL}$ streptomycin, which was replenished everyday. The ucOC concentration in WT adult mice is approximately $7 \mathrm{ng} / \mathrm{mL}$. Thus, in these experiments, we used amounts of ucOC ranging from 50 -fold lower than its physiological concentration to 7 -fold higher. Uncarboxylated osteocalcin (AnaSpec Inc, Fremont, CA, USA) was resuspended and diluted in phosphate-buffered saline (PBS) to obtain final concentrations of $0.1-50 \mathrm{ng} / \mathrm{mL}$. To examine the effect of ucOC on cell proliferation and differentiation, the medium was replaced with serum-free medium or with DM containing ucOC (0-50ng/ $\mathrm{mL}$ ) at the indicated times. In some experiments, $\mathrm{C} 2 \mathrm{C} 12$ cells were pretreated with the phosphatidylinositol3-kinase (PI3K) inhibitor wortmannin (SB203580 or U0126, Sigma, St. Louis, MO, USA) 30 min prior to ucOC stimulation. GPRC6A siRNAs were obtained from Santa Cruz (Santa Cruz Biotechnology Inc., California, USA), and C2C12 cells were transfected with the siRNAs using Lipofectamine 2000 (Invitrogen, Carlsbad, CA, USA) according to the manufacturer's protocol.

\section{Growth curves}

C2C12 myoblasts were plated in 96-well plates at 10, 000-12, 000 cells per well. Twenty-four hours later, the culture medium was changed to serum-free medium containing varying doses of ucOC $(0 \mathrm{ng} / \mathrm{mL}$, $0.1 \mathrm{ng} / \mathrm{mL}, 1 \mathrm{ng} / \mathrm{mL}, 10 \mathrm{ng} / \mathrm{mL}$ or $50 \mathrm{ng} / \mathrm{mL}$ ). The experiment was continued until the control cells reached subconfluence ( 72 hours). The cells were counted using the CCK8 assay, and the average cell counts for each day were used to plot the growth curves of the ucOC-treated myoblasts.

\section{Cell proliferation assay}

At the indicated times, C2C12 cells were treated with CCK8 (10 $\mu \mathrm{L} /$ well, Sigma) for an additional 1 h. Finally, we recorded the absorbance at $450 \mathrm{~nm}$ using a microplate absorbance reader (Tecan, Safire II, Switzerland). The OD value (absorbance at $450 \mathrm{~nm}$ ) was detected using an enzyme-linked immunosorbent assay (ELISA) (BIO-RAD, USA).

\section{Immunohistological staining}

Growing adherent cells on coverslips were obtained each day and fixed in $4 \%$ formaldehyde. The fixed cells were incubated overnight at $4^{\circ} \mathrm{C}$ with a primary antibody against PCNA (cell proliferation marker, 1:100; Santa Cruz Biotechnology). The next day, biotin-labelled secondary antibodies were applied and were detected with diaminobenzidine (Sigma). Then, nuclei were stained with haematoxylin (Sigma). PCNApositive nuclei were stained brown, and PCNA-negative nuclei were stained blue. The proportion of PCNApositive nuclei was determined through image analysis of the cells. Photomicrographs were captured and analysed using Image-Pro-Plus 5.0 software (Media Cybernetics, Silver Spring, MD, USA). The area of PCNApositive nuclei per high-power field was calculated. 
Liu et al.: Osteocalcin Induces Proliferation and Promotes Differentiation in $\mathrm{C} 2 \mathrm{C} 12$ Myoblast Cells

\section{Differentiation experiments}

C2C12 cells were treated with ucOC at $0 \mathrm{ng} / \mathrm{mL}, 0.1 \mathrm{ng} / \mathrm{mL}, 1 \mathrm{ng} / \mathrm{mL}, 10 \mathrm{ng} / \mathrm{mL}$, and $50 \mathrm{ng} / \mathrm{mL}$ in DM. The medium was exchanged daily for fresh DM containing ucOC. For morphological analysis, the cells were fixed with methanol/acetone (Sigma), and the number of multinucleated myotubes present in a field (1 $\mu \mathrm{m} 2$ $\times 100$ ) was counted using a phase-contrast microscope (OLYMPUS, CX41, Tokyo) $72 \mathrm{~h}$ after switching to DM.

\section{Western Blotting}

Cells were lysed for 30 min on ice in radioimmunoprecipitationassay (RIPA) lysis buffer (Solarbio, Beijing, China) containing $0.1 \mathrm{mM}$ PMSF and a protease inhibitor (Roche).The samples were subjected to $12 \%$ SDSPAGE and transferred to nitrocellulose membranes. Blots were probed using primary antibodies, including anti-phospho-Akt (Cell Signaling Technology, CST, Boston, MA, USA), anti-Akt (CST), anti-phospho-P38 (CST), anti-P38 (CST), anti-phospho-ERK1/2 (CST), anti-ERK1/2 (CST), anti-GPRC6A (SantaCruz), anti-MyHC (Santa Cruz), and anti-GAPDH (CST). The lysates were subjected to standard Western Blotting analysis. The images were obtained usingTanon-5200 (Tanon, Shanghai, China). The relative densities of the specific bands were quantified using the image detection software Image Lab 5.0 (Bio-Rad Laboratories Inc., California, USA).

\section{Short interfering RNA (siRNA) and transfection}

siRNA transfection was used to downregulate GPRC6A expression. All the siRNAs were purchased from Santa Cruz Biotech (Santa Cruz, CA, USA). The GPRC6A-targeted siRNA (si GPRC6A) and control siRNA (si Ctrl) were dissolved separately in Optimem I (Invitrogen). After 10 min of equilibration at room temperature, each RNA solution was combined with an equal volume of Lipofectamine ${ }^{\mathrm{TM}} 2000$ solution, mixed gently, and allowed to form siRNA liposomes for $20 \mathrm{~min}$.

\section{Isolation of RNA and RT-PCR analysis}

Total RNA was extracted from C2C12 cells using a Nucleo SpinRNA kit (TakaraBio, Shiga, Japan) according to the manufacturer's instructions. Isolated total RNA (100ng) was subjected to reverse transcription with or without Prime Script Reverse Transcriptase (Takara Bio) and PCR analysis using primers for GPRC6A, 5'-CCAGACGACCACAAATCCAG- 3' and 5'-GATTCATAACTCACCTGTGGC-3', and for GAPDH, 5'ACCACAGTCCATGCCATCAC-3'and 5'-TCCACCACCCTGTTGCTGTA-3'.

\section{Statistical analysis}

Statistical analysis was performed using GraphPad Prism 6.0 (GraphPad Software, Inc., La Jolla, CA, USA). Unless indicated otherwise, the data are presented as the mean \pm S.E.M. Comparison between two groups was analysed using Student's t-test. One-way ANOVA was utilized for multiple comparisons followed by Tukey's post hoc test. A p value $<0.05$ was defined as significant.

\section{Results}

ucOC induces proliferation of myoblasts

In the proliferative phase, we analysed the effect of ucOC on $\mathrm{C} 2 \mathrm{C} 12$ cell proliferation. Serum-deprived C2C12 cells were incubated with GM or with various concentrations of ucOC (0-50ng/mL). Morphological changes in the C2C12 cells and in the areas of PCNApositive cells were evaluated using immunohistological analysis. Cells treated with ucOC showed increased proliferation and a phenotype very similar to that of cells treated with GM (Fig. 1A and 1B), although treatment with standard GM was much more effective. We found that enlargement of the area of PCNA-positive cells occurred at ucOC concentrations as low as $0.1 \mathrm{ng} / \mathrm{mL}$. At $10 \mathrm{ng} / \mathrm{mL}$, ucOC triggered up to a 1.36-fold increase in the area of PCNApositive cells. At a concentration of $50 \mathrm{ng} / \mathrm{mL}$, the effect of ucOC was reduced (Fig. 1B).

We further studied the stimulatory effect of ucOC on C2C12 cell growth using a CCK8 assay. The average cell counts for each day were used to plot a growth curve (Fig. 1C). The results indicated that in the presence of ucOC the number of cells increased at 24, 48, and 72 h. The results demonstrate that ucOC induces $\mathrm{C} 2 \mathrm{C} 12$ cell proliferation with a dose-response 
Liu et al.: Osteocalcin Induces Proliferation and Promotes Differentiation in $\mathrm{C} 2 \mathrm{C} 12$ Myoblast Cells

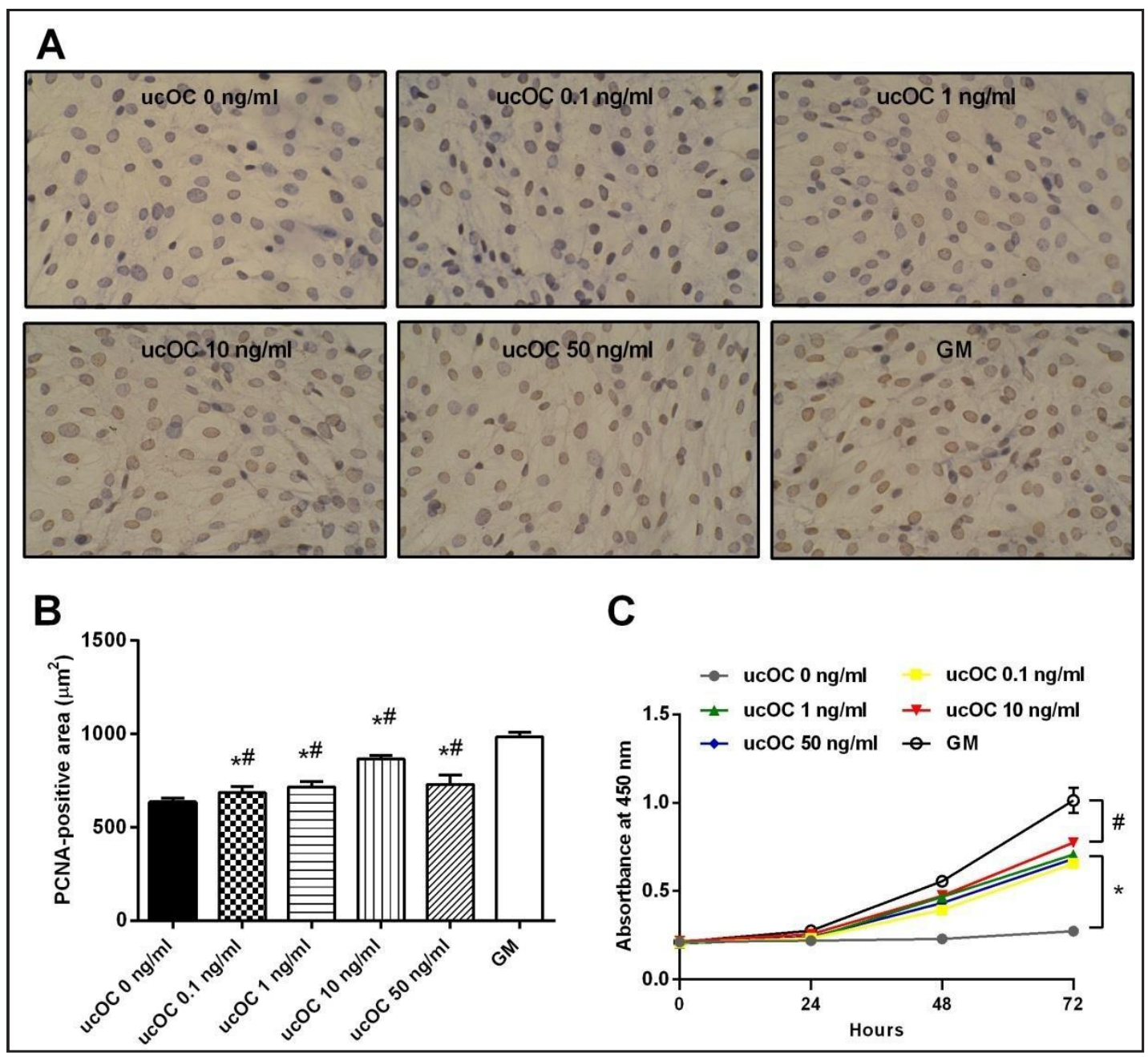

Fig. 1. ucOC induces proliferation of C2C12 cells in vitro. (A), (B) Immunohistochemical analysis of PCNA in C2C12 cells. (A) The proportion of PCNA-positive nuclei and (B) the calculated area of PCNA-positive nuclei were significantly higher in the ucOC $(0.1-50 \mathrm{ng} / \mathrm{ml})$ groups than in the control group (ucOC $0 \mathrm{ng} / \mathrm{ml}$ group). (C) Growth curves result represent the effects of ucOC on cell growth in $\mathrm{C} 2 \mathrm{C} 12$. The absorbance value was detected at 24, 48, and 72 hours. Data are representative of three independent experiments performed in triplicate. ${ }^{*} \mathrm{P}<0.05$ versus control group, $\# \mathrm{P}<0.05$ versus $\mathrm{GM}$ group.

relationship similar to that observed for the area of PCNA-positive cells. Thus, these data show that low concentrations of ucOC act as a powerful regulator of $\mathrm{C} 2 \mathrm{C} 12$ proliferation.

\section{PI3K/Akt and p38 MAPK signalling are involved in ucOC-mediated priming of myoblast} proliferation

To examine the molecular mechanisms responsible for the proliferative effects of ucOC in myoblasts, we evaluated ucOC-dependent activation of the ERK1/2, PI3K/Akt and P38 MAPK pathways. These pathways play major roles in regulating cell growth, survival, and differentiation. C2C12 cells were serum-deprived for $24 \mathrm{~h}$ and then treated with $10 \mathrm{ng} / \mathrm{mL}$ $\mathrm{ucOC}$ for various times $(10 \mathrm{~min}, 6 \mathrm{~h}$, and $24 \mathrm{~h}$ ). We found that ucOC treatment significantly increased the levels of p-Akt and p-P38 MAPK in the cells at 24h (Fig. 2A).

To address the role of Akt in ucOC-induced cell proliferation, C2C12 cells were pretreated with wortmannin, a reversible inhibitor of Akt. After wortmannin treatment, the phosphorylation status of Akt was attenuated. Accordingly, C2C12 cell proliferation

\section{KARGER}




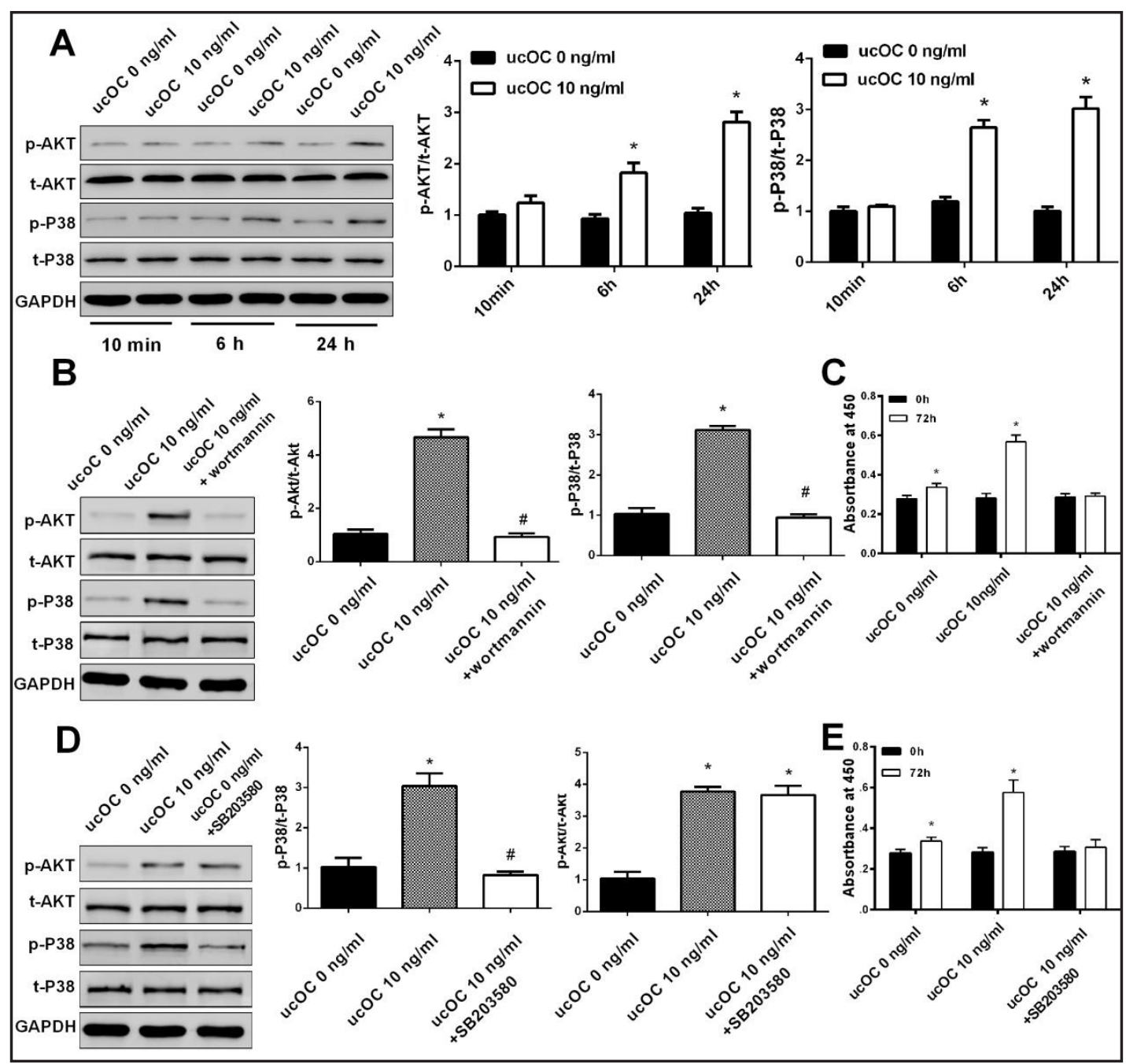

Fig. 2. PI3K/Akt and P38 MAPK signalling are involved in ucOC-mediated priming of C2C12 cells proliferation. (A) The expression of p-Akt, t-Akt, p-P38, t-P38 and GAPDH was detected in C2C12 cells by Western Blotting analyses. (B), (C) Wortmannin reduced Akt and P38 MAPK phosphorylation, inhibited the effect of ucOC on C2C12 cells proliferation. (D), (E) SB203580 reduced P38 MAPK phosphorylation, inhibited the effect of ucOC on C2C12 cells proliferation. (B), (D) Phosphorylation levels of Akt and P38 MAPK were determined by Western Blotting analysis at 24 hours. (C), (E) C2C12 cells were counted using CCK8 assay at 72 hours. GAPDH was used as an internal control. Data are representative of three independent experiments performed in triplicate. ${ }^{*} \mathrm{P}<0.05$ versus control group (ucOC $0 \mathrm{ng} / \mathrm{ml}$ group), $\# \mathrm{P}<0.05$ versus ucOC $10 \mathrm{ng} /$ ml group.

significantly decreased by 50\% (Fig. 2B and 2C). We then tested whether P38 MAPK might also be involved in this pathway. As shown in Fig. 2D and 2E, inhibition of P38 MAPK by SB203580 also significantly inhibited the effect of ucOC on myoblast proliferation. In addition, wortmannin pretreatment reduced P38 MAPK phosphorylation. In contrast, SB203580 did not affect Akt activation, suggesting that P38 MAPK is a downstream signalling factor of Akt. However, no significant difference was detected in the level of p-ERK between groups following ucOC stimulation (data not shown). Taken together, these results suggest that ucOC stimulates the priming of myoblast proliferation through the sequential activation of Akt and p38 MAPK. 
Liu et al.: Osteocalcin Induces Proliferation and Promotes Differentiation in $\mathrm{C} 2 \mathrm{C} 12$ Myoblast Cells

ucOC-induced myoblast proliferation is blocked by GPRC6A knockdown

We initially examined whether myoblasts express receptors for ucOC. mRNA analysis by qRT-PCR revealed that myoblasts express GPRC6A mRNA in a time-dependent manner during ucOC treatment, and this was confirmed by Western Blotting analysis (Fig. 3A). We speculated that GPRC6A would be stimulated by ucOC to activate Akt and P38 MAPK. Therefore, we used siRNA to knockdown GPRC6A to investigate the activation of Akt and P38 MAPK (Fig. 3B). The activation of Akt and P38 MAPK was inhibited by the knockdown of GPRC6A (Fig. 3C). Accordingly, C2C12 cell proliferation significantly decreased by 51\% (Fig. 3D). In conclusion, we believe that GPRC6A functions as the receptor of ucOC and that it acts in the upper portion of the Akt and P38 MAPK signalling pathways to regulate myoblast proliferation.

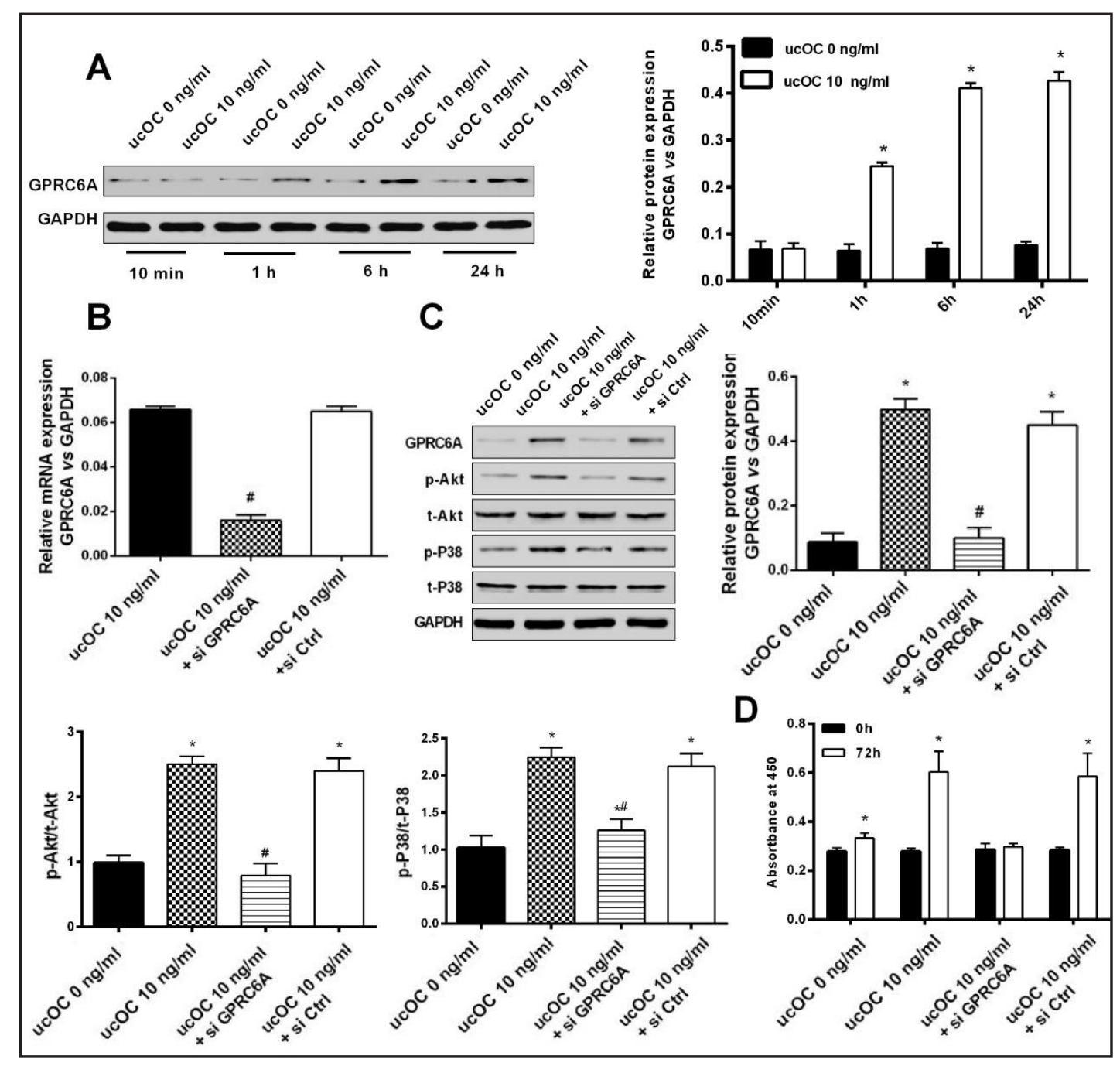

Fig. 3. GPRC6A knockdown blocks ucOC-induced myoblast proliferation in C2C12 cells. (A) Western Blotting analysis revealed that the expression of GPRC6A is time-dependent following ucOC treatment in C2C12 cells. (B) qRT-PCR suggested that the expression of GPRC6A gene was down-regulated by GPRC6A siRNA transfection in C2C12 cells. (C) The expression of GPRC6A protein, phosphorylation levels of Akt and P38 MAPK were determined by Western Blotting analysis at 24 hours. (D) C2C12 cells were counted using CCK8 assay at 72 hours. GAPDH was used as an internal control. Data are representative of three independent experiments performed in triplicate. ${ }^{*} \mathrm{P}<0.05$ versus control group (ucOC $0 \mathrm{ng} / \mathrm{ml}$ group), $\# \mathrm{P}<0.05$ versus ucOC $10 \mathrm{ng} / \mathrm{ml}$ group. 
Liu et al.: Osteocalcin Induces Proliferation and Promotes Differentiation in $\mathrm{C} 2 \mathrm{C} 12$ Myoblast Cells

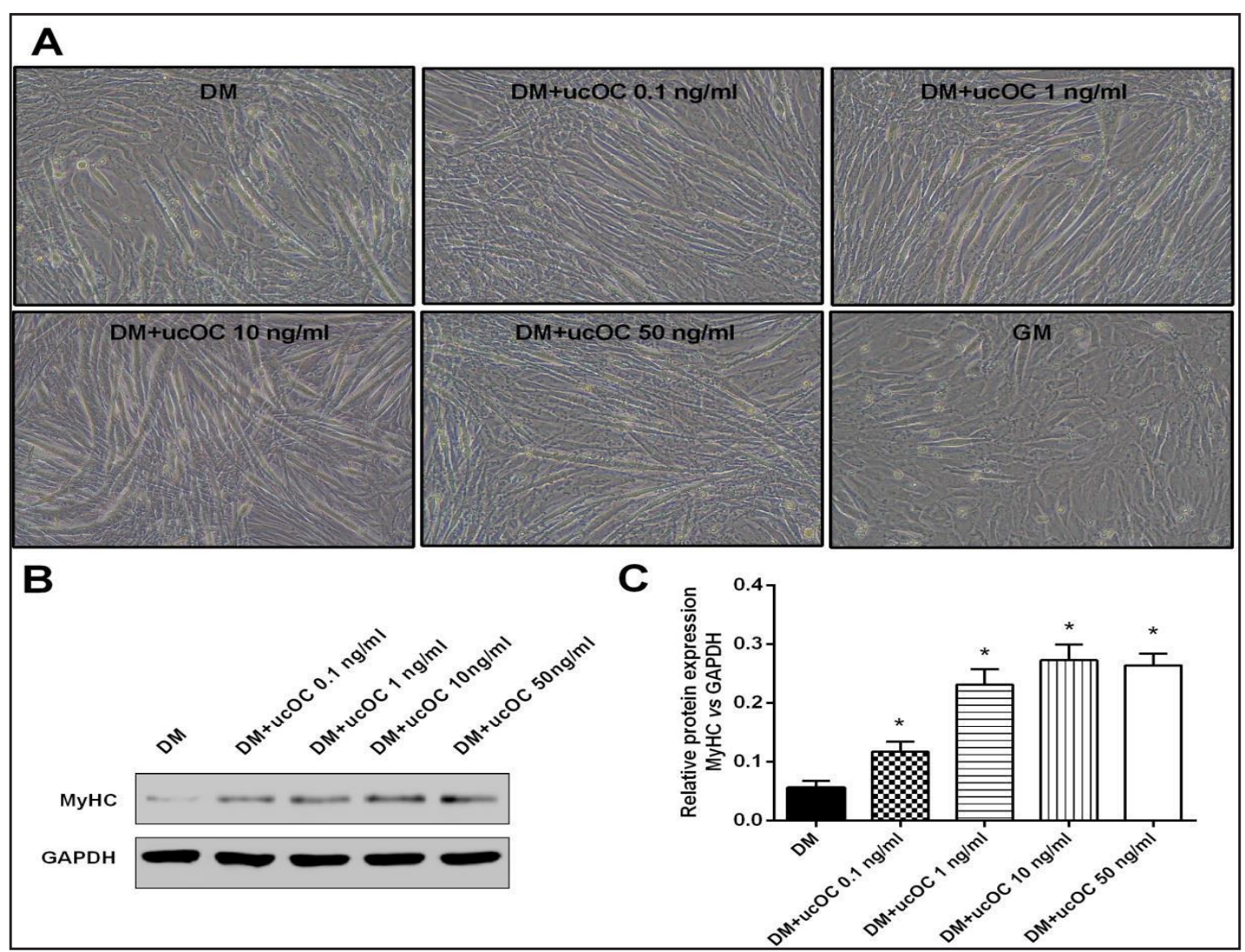

Fig. 4. ucOC enhances myogenic differentiation in C2C12 cells. (A) Representative views of the morphological changes of differentiated C2C12 cells at 72 hours. (B) Protein levels of muscle-specific protein MyHC was determined by Western Blot analysis at 24 hours. GAPDH was used as an internal control. Data are representative of three independent experiments performed in triplicate. ${ }^{*} \mathrm{P}<0.05$ versus control group (DM group).

\section{ucOC enhances the differentiation of myoblasts}

The C2C12 cell line is commonly used as an in vitro model for myogenesis. To examine the effect of ucOC on myogenic differentiation, $\mathrm{C} 2 \mathrm{C} 12$ myoblasts were induced to differentiate by switching the cells to DM in the presence of various concentrations of ucOC $(0-50 \mathrm{ng} / \mathrm{mL})$ for 72 hours. We observed that the addition of ucOC to the DM resulted in the formation of larger myotubes with more nuclei per myotube in comparison to cells treated with standard DM (Fig. 4A). In addition, the expression of the muscle-specific protein MyHC was significantly upregulated by 2.13-, 4.0-, 5.0-, and 4.5-fold compared with that of the control by treatment with ucOC at $0.1,1,10$, and $50 \mathrm{ng} / \mathrm{mL}$, respectively (Fig. 4B). Taken together, these data indicate that ucOC treatment results in a statistically significant promotion of C2C12 cell differentiation at themorphological as well as at the biochemical level.

\section{Involvement of GPRC6A-ERK1/2 signalling in ucOC-mediated myogenic differentiation}

To confirm the involvement of ucOC in the process of myogenesis, we treated $\mathrm{C} 2 \mathrm{C} 12$ cells with $10 \mathrm{ng} / \mathrm{mL}$ ucOC and assessed the activation levels of the PI3K/Akt and P38 MAPK pathways, which our data show are involved in the ucOC-induced priming of myoblast proliferation. After ucOC treatment, the phosphorylation levels of these proteins remained almost unchanged (data not shown). We then analysed the ERK1/2 pathway, another wellknown signalling pathway that has been analysed in many muscle studies by Western Blotting analysis using an antibody that recognizes an active, phosphorylated form of this kinase. The results confirmed that ucOC treatment significantly increased the levels of p-ERK1/2, whereas the total protein levels remained relatively constant (Fig. 5A). We

\section{KARGER}




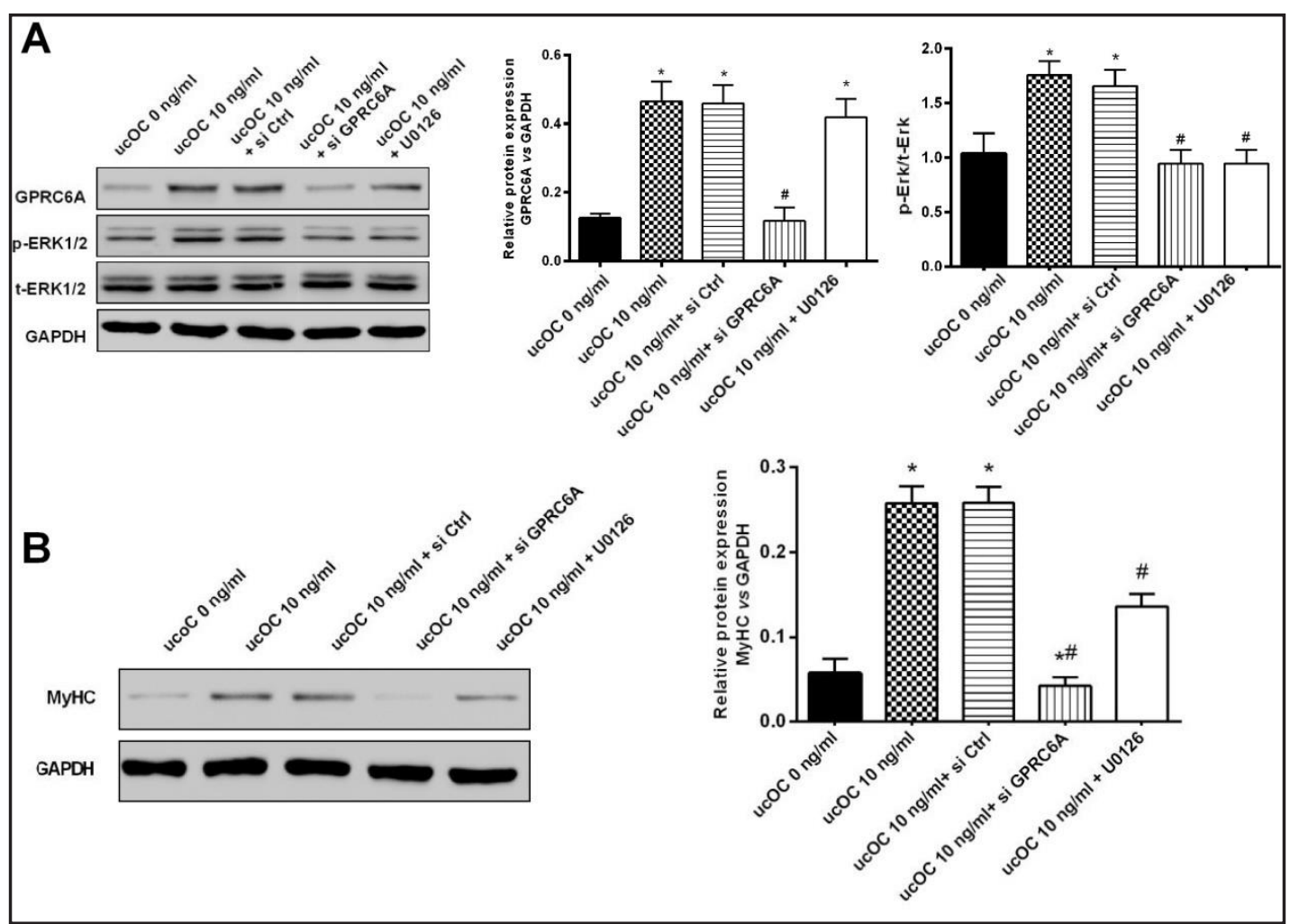

Fig. 5. GPRC6A-ERK1/2 signaling are involved in ucOC-mediated myogenic differentiation in C2C12 cells. (A), (B) C2C12 cells were transfected with GPRC6A siRNA or pretreated with ERK1/2 inhibitor U0126 respectively. Western Blotting analysis was performed to examine GPRC6A expression (A), phosphorylation level of ERK1/2 (A) and MyHC expression (B). GAPDH was used as an internal control. Data are representative of three independent experiments performed in triplicate. ${ }^{*} \mathrm{P}<0.05$ versus control group (ucOC $0 \mathrm{ng} / \mathrm{ml}$ group), $\# \mathrm{P}<0.05$ versus ucOC $10 \mathrm{ng} / \mathrm{ml}$ group.

next assessed whether ucOC treatment overrides the block of myoblast differentiation by inhibition of ERK1/2. C2C12 cells were treated with the pharmacological inhibitor U0126 for 30 min prior to the addition of ucOC and then allowed to differentiate for $24 \mathrm{~h}$, followed by Western Blotting analysis. As predicted, treatment with U0126 reduced the phosphorylation of ERK1/2 and decreased the expression of MyHC (Fig. 5B). In conclusion, our results demonstrate that ucOC accelerates myoblast differentiation at least partially through the activation of ERK1/2 signalling.

Next, we used siRNA to knockdown GPRC6A expression to investigate the activation of ERK1/2. The activation of ERK1/2 was inhibited due to the knockdown of GPRC6A (Fig. 5A). On the other hand, inhibition of ERK1/2 by U0126 did not affect GPRC6A. To confirm the involvement of GPRC6A in myogenic differentiation caused by ucOC, we examined the ucOCinduced expression of MyHC in the presence of siRNA for GPRC6A. The results demonstrated significant inhibition of the expression of MyHC in the presence of GPRC6A siRNA (Fig 5B). Thus, our data demonstrate that inhibition of GPRC6A blocks ucOC-induced myoblast differentiation and suggest that GPRC6A-ERK1/2 signalling pathways are involved in ucOCstimulated myoblast differentiation.

\section{Discussion}

Sarcopenia, a syndrome characterized by progressive and generalized loss of skeletal muscle mass and function, results in frailty, increased vulnerability to adverse health 
outcomes, and decreased quality of life in the elderly population [31]. Several mechanisms may be involved in the onset and progression of sarcopenia. These mechanisms include protein synthesis, proteolysis, neuromuscular integrity and musculoskeletal coordination [32-35]. In the present study, we identified a novel role of the bone-derived hormone osteocalcin in musculoskeletal coordination. We showed that exogenous ucOC is sufficient to induce myoblast proliferation and enhance myogenic differentiation in vitro and that the ability of ucOC to induce myoblast proliferation depends on activation of the PI3K/Akt and p38MAPK pathways. In addition, GPRC6A-ERK1/2 signalling was shown to be involved in ucOC-mediated myogenic differentiation, and knockdown of GPRC6A by siRNA markedly attenuated the effects of ucOC.

Neither bone nor muscle tissue was previously suspected to perform endocrine functions, let alone to affect so many physiological processes in a significant manner [23]. Osteocalcin, the most abundant non-collagenous bone matrix protein, is a small g-carboxyglutamate protein that is preferentially expressed by osteoblasts and that regulates bone mineralization [19]. Accumulating results have implicated ucOC in glucose metabolism [20, 36]. Because skeletal muscle is a major site of glucose disposal [37], this suggests that the ucOC may be linked directly or indirectly to skeletal muscle mass and strength. At present, there is little evidence from animal experiments to show that ucOC is related to muscle mass. Paula et al. [25]. reported that osteocalcin signalling in myofibres is necessary to maintain muscle mass in older mice in part because it promotes protein synthesis in myotubes. Hua et al. [26]. indicated that connexin43 ( $\mathrm{Cx} 43$ ), a gap junction protein that transduces mechanical signals in osteoblasts/osteocytes, indirectly modulates skeletal muscle growth and function, potentially via an endocrine effect of ucOC. Furthermore, another recent study showed that loss of muscle mass and strength under atrophic conditions induced by hindlimb immobilization is related to a reduction in ucOC levels in rats [38]. However, the mechanisms involved in the regulation of biological processes in muscle are poorly understood. In this study, we identified a novel function of ucOC and showed that it directly coordinates myoblast proliferation and the myogenic differentiation programme in vitro, suggesting that ucOC possesses myoblast proliferation and differentiation promoting effects under various conditions. Consistent with this, osteocalcin has been shown to induce proliferation of pancreatic beta cells and to stimulatethe differentiation of fat cells [24, 39].

MAPK signalling plays an important role in complex cellular processes such as proliferation, differentiation and survival [40]. At least 3 MAPK families have been characterized: ERK1/2, P38 MAPK and Jun kinase [41]. In addition to MAPK, PI3K/Akt is also an important signalling factor for cell survival and growth $[42,43]$. In this study, we report a new mechanism by which ucOC stimulates myoblast proliferation via sequential activation of the Akt and P38 MAPK pathways. Using chemical inhibitors of Akt and P38 MAPK, we revealed that Akt and P38 MAPK are sequentially activated. During the differentiation phase, ucOC treatment enhanced the phosphorylation of ERK1/2, the key myogenic kinase that activates MyHC. The enhanced myoblast differentiation caused by ucOC treatment can be decreased by inhibition of ERK1/2 by U0126. Moreover, knockdown of GPRC6A by siRNA inhibited Akt, P38 MAPK and ERK1/2 activation and attenuated ucOC-induced myoblast proliferation and myogenic differentiation in C2C12 cells.

In general, ERK1/2 is essential for muscle cell proliferation, whereas the PI3K/Akt pathway mainly promotes differentiation of those cells $[44,45]$. However, the findings of Yang et al. and of our team are inconsistent with these general ideas [46, 47]. The conflicting results may be due to differences in the experimental set ups used. In addition, because ucOC activates both ERK1/2 and Akt signalling pathways in C2C12 cells, we hypothesized that the ERK1/2 and Akt signaling pathways might interact with each other. It is also possible that ucOC stimulates a more complicated signaling network. Further research to test this hypothesis is warranted.

Myoblast proliferation and differentiation are early critical processes that regulate muscle growth and regeneration. Defects in satellite cell proliferation or differentiation are associated with a variety of muscular diseases. In the current study, we utilized a commonly 
used in vitro model of muscle cells to study the mechanism of action of ucOC in muscle cells. We provide evidence supporting a role for ucOC as a factor that regulates the balance between muscle cell proliferation and differentiation by modulating signalling pathways. Our data identify ucOC as a potential therapeutic target for alleviating the reduction in muscle mass associated with muscle disorders and ageing. To the best of our knowledge, only a few human studies have investigated osteocalcin in relation to muscle mass and function, and these studies have led to inconsistent conclusions [48-50]; thus, the role of ucOC in muscle mass and physical function in humans is still unknown. Further research in humans is needed to confirm the data obtained inanimal studies.

In conclusion, our results provide the first evidence that osteocalcin induces proliferation via positive activation of the PI3K/Akt and P38 MAPK pathways and that it promotes differentiation through activation of the GPRC6A-ERK1/2 pathway. Age-related sarcopenia implies progressive deterioration of the molecular mechanisms responsible for cell viability, proliferation and differentiation. We believe that our data support the idea that osteocalcin plays a major role in sarcopenia by promoting myoblast cellular function. However, further investigation is required to translate these findings from in vitro experiments to humans. The salient findings from the present study provide us with a better understanding of osteocalcin's functions and offer exciting opportunities for the development of new therapeutic strategies for muscle diseases such as sarcopenia.

\section{Acknowledgements}

This work was supported by grants from the National Natural Science Foundation of China (grant No. 81272203) and the Natural Science Foundation of Fujian Province of China (grant No. 2016J01624).

\section{Disclosure Statement}

The authors declare no Disclosure Statement.

\section{References}

1 Cruz-Jentoft AJ, Baeyens JP, Bauer JM, Boirie Y, Cederholm T, Landi F, Martin FC, Michel J-P, Rolland Y, Schneider SM: Sarcopenia: European consensus on definition and diagnosis Report of the European Working Group on Sarcopenia in Older People. Age Ageing 2010;39:412-423.

2 Woo J, Leung J, Morley J: Defining sarcopenia in terms of incident adverse outcomes. J Am Med Dir Assoc 2015;16:247-252.

-3 Cawthon PM, Blackwell TL, Cauley J, Kado DM, Barrett-Connor E, Lee CG, Hoffman AR, Nevitt M, Stefanick ML, Lane NE: Evaluation of the usefulness of consensus definitions of sarcopenia in older men: results from the Observational Osteoporotic Fractures in Men Cohort Study. J Am Geriatr Soc 2015;63: 22472259.

-4 Perna S, Peroni G, Anna FM, Bartolo A, Naso M, Miccono A, Rondanelli M: Sarcopenia and sarcopenic obesity in comparison: prevalence, metabolic profile, and key differences. A cross-sectional study in Italian hospitalized elderly. Aging Clin Exp Res DOI: 10.1007/s40520-016-0701-8.

5 Wang YJ, Wang Y, Zhan JK, Tang ZY, He JY, Tan P, Deng HQ Huang W, Liu YS: Sarco-Osteoporosis: Prevalence and Association with Frailty in Chinese Community-Dwelling Older Adults. Int J Endocrinol DOI: $10.1155 / 2015 / 482940$.

6 Michael D, Cornel CS, Thomas B, Jürgen MB, Ralf S, The FiAT intervention group: Osteosarcopenia is more than sarcopenia and osteopenia alone. Aging Clin Exp Res 2016;28:895-899.

7 Camille T, YohannW, Marie-Jeanne D, Stéphane W, Véronique C: Muscle and bone, two i nterconnected tissues. Ageing Res Rev 2015;21:55-70. 
Liu et al.: Osteocalcin Induces Proliferation and Promotes Differentiation in C2C12 Myoblast Cells

8 Gerard K, Eric NO: Bone and Muscle Endocrine Functions: Unexpected Paradigms of Inter-organ Communication. Cell 2016;164:1248-1256.

- Luisella C, Maria LB: Muscle-bone interactions: basic and clinical aspects. Endocrine DOI: 10.1007/s12020013-0026-8.

10 Scimeca M, Piccirilli E, Mastrangeli F, Rao C, Feola M, Orlandi A, Gasbarra E, Bonanno E: Tarantino U: Bone Morphogenetic Proteins and myostatin pathways: key mediator of human sarcopenia. J Transl Med 2017;15:34-44.

-11 Ambrogini E, Almeida M, Martin-Millan M, Paik JH, Depinho RA, Han L, Goellner J, Weinstein RS, Jilka RL, O'Brien CA, Manolagas SC: FoxO-mediated defense against oxidative stress in osteoblasts is indispensable for skeletal homeostasis in mice Cell Metab 2011;11:136-146.

12 Guo B, Zhang ZK, Liang C, Li J, Liu J, Lu A, Zhang BT, Zhang G: Molecular Communication from Skeletal Muscle to Bone: A Review for Muscle-Derived Myokines Regulating Bone Metabolism. Calcified Tissue International 2017;100:184-192.

13 Bikle DD, Tahimic C, Chang W, Wang Y, Philippou A, Barton ER: Role of IGF-I signaling in muscle bone interactions. Bone 2015;80:79-88.

14 Armakolas N, Armakolas A, Antonopoulos A, Dimakakos A, Stathaki M, Koutsilieris M: The role of the IGF-1 Ec in myoskeletal system and osteosarcoma pathophysiology. Crit Rev Oncol Hematol 2016;108:137-145.

15 Mo C, Zhao R, Vallejo J, Igwe O, Bonewald L, Wetmore L, Brotto M: Prostaglandin E2 promotes proliferation of skeletal muscle myoblasts via EP4 receptor activation. Cell Cycle 2015;14:1507-1516.

16 Pedersen BK, Febbraio MA: Muscles, exercise and obesity: skeletal muscle as a secretory organ. Nat Rev Endocrinol 2012;8:457-465.

17 Mera P, Laue K, Ferron M, Confavreux C, Wei J, Galán-Díez M, Lacampagne A, Mitchell SJ, Mattison JA, Chen Y, Bacchetta J, Szulc P, Kitsis RN, de Cabo R, Friedman RA, Torsitano C, McGraw TE, Puchowicz M, Kurland I, Karsenty G: Osteocalcin Signaling in Myofibers Is Necessary and Sufficient for Optimum Adaptation to Exercise. Cell Metabolism 2016;23:1078-1092.

18 Lynda FB,1 Douglas PK, Thomas LC, Karyn E, Eric SO, Regis JO'K, Roger AF: Forum on Bone and Skeletal Muscle Interactions: Summary of the Proceedings of an ASBMR Workshop. Journal of Bone and Mineral Research 2013;28:1857-1865.

19 Huang J, Meng Y, Liu Y, Chen Y, Yang H, Chen D, Shi J, Guo Y. MicroRNA-320a Regulates the Osteogenic Differentiation of Human Bone Marrow-Derived Mesenchymal Stem Cells by Targeting HOXA10. Cell Physiol Biochem. 2016;38(1):40-8.

20 Lee NK, Sowa H, Hinoi E, Ferron M, Ahn JD, Confavreux C, Dacquin R, Mee PJ, McKee MD, Jung DY, Zhang Z, Kim JK, Mauvais-Jarvis F, Ducy P, Karsenty G: Endocrine Regulation of Energy Metabolism by the Skeleton. Cell 2007;130:456-469.

-21 Franck O, Grzegorz S, Olga S, Mathieu F, Chang H, Charles ES, Louis H, Susan S, Bryan LR, Patricia D, Gerard K: Endocrine Regulation of Male Fertility by the Skeleton. Cell 2011;144:796-809.

-22 Booth FW, Ruegsegger GN, Olver TD: Exercise Has a Bone to Pick with Skeletal Muscle. Cell Metab 2016;23:961-962.

-23 Zhou H, Seibel MJ: Bone: Osteoblasts and global energy metabolism-beyond osteocalcin. Nat Rev Rheumatol DOI: 10.1038/nrrheum.2017.35.

24 Meredith LZ, Thomas LC, Ryan CR: New insights into the biology of osteocalcin. Bone 2016;82:42-49.

25 Paula M, Kathrin L, Wei J, Julian MB, Gerard K: Osteocalcin is necessary and sufficient to maintain muscle mass in older mice. Mol Metab 2016;5:1042-1047.

26 Shen H, Susan G, Roberto C, Stavros T: Deletion of connexin43 in osteoblasts/osteocytes leads to impaired muscle formation in mice. J Bone Miner Res 2015;30:596-605.

-27 Jørgensen S, Have CT, Underwood CR, Johansen LD, Wellendorph P, Gjesing AP, Jørgensen CV, Quan S, Rui G, Inoue A, Linneberg A,Grarup N, Jun W, Pedersen O, Hansen T, Bräuner-Osborne H: Genetic Variations in the Human G Protein-coupled Receptor Class C, Group 6, Member A (GPRC6A) Control Cell Surface Expression and Function. J Biol Chem 2017;292:1524-1534.

-28 Pi M, Kapoor K, Ye R, Nishimoto SK, Smith JC, Baudry J, Quarles LD: Evidence for Osteocalcin Binding and Activation of GPRC6A in $\beta$-Cells. Endocrinology 2016;157:1866-1880.

-29 Oury F, Ferron M, Huizhen W, Confavreux C, Xu L, Lacombe J, Srinivas P, Chamouni A, Lugani F, Lejeune H, Kumar TR, Plotton I, Karsenty G: Osteocalcin regulates murine and human fertility through a pancreasbone-testis axis. J Clin Invest 2013;123:2421-2433. 
Liu et al.: Osteocalcin Induces Proliferation and Promotes Differentiation in $\mathrm{C} 2 \mathrm{C} 12$ Myoblast Cells

-30 Mizokami A, Yasutake Y, Higashi S, Kawakubo-Yasukochi T, Chishaki S, Takahashi I, Takeuchi H, Hirata M: Oral administration of osteocalcin improves glucose utilization by stimulating glucagon-like peptide-1 secretion. Bone 2014;69:68-79.

-31 Chen LK, Liu LK, Jean W, Prasert A, Tung-Wai A, Kamaruzzaman SB, Ming-Yueh C, Chen LY, Pi-Shan H, Orapitchaya K, Jenny S.W. L, Wei-Ju L, Yunhwan L, Chih-Kuang L, Panita L, Lin CS, Peng LN, Shosuke S,Takao S, Chang Won W, Chih-Hsing W, Si-Nan W, Zhang T, Zeng P, Masahiro A, Hidenori A: Sarcopenia in Asia: Consensus Report of the Asian Working Group for Sarcopenia. JAMDA 2014;15:95-101.

-32 Gumucio JP, Mendias CL: Atrogin-1, MuRF-1, and sarcopenia. Endocrine 2013;43:12-21.

-33 Girón MD, Vílchez JD, Salto R, Manzano M, Sevillano N, Campos N, Argilés JM, Rueda R, López-Pedrosa JM: Conversion of leucine to $\beta$-hydroxy- $\beta$-methylbutyrate by $\alpha$-keto isocaproate dioxygenase is required for a potent stimulation of protein synthesis in L6 rat myotubes. J Cachexia Sarcopenia Muscle 2016;7:68-78.

34 Thapaliya S, Runkana A, McMullen MR, Nagy LE, McDonald C, Naga Prasad SV, Dasarathy S: Alcohol-induced autophagy contributes to loss in skeletal muscle mass. Autophagy 2014;10:677-690.

-35 Clark DJ, Patten C, Reid KF, Carabello RJ, Phillips EM, Fielding RA: Impaired voluntary neuromuscular activation limits muscle power in mobility-limited older adults. J Gerontol A Biol Sci Med Sci 2010;65:495502.

36 Mizokami A, Kawakubo-Yasukochi T, Hirata M: Osteocalcin and its endocrine functions. Biochem Pharmacol DOI: 2017.02.001.

-37 Josep MA, Nefertiti C, José M. Lopez-P, Ricardo R, Leocadio Rodriguez M: Skeletal Muscle Regulates Metabolism via Interorgan Crosstalk: Roles in Health and Disease. JAMDA 2016;17:789-796.

-38 Lin X, Hanson E, Betik AC, Brennan-Speranza TC, Hayes A, Levinger I: Hindlimb Immobilization, But Not Castration, Induces Reduction of Undercarboxylated Osteocalcin Associated With Muscle Atrophy in Rats. J Bone Miner Res 2016;31:1967-1978.

-39 Zhang Y, Zhou P, Kimondo JW: Adiponectin and osteocalcin: relation to insulin sensitivity. Biochem Cell Biol 2012;90:613-620.

40 Chang L, Karin M: Mammalian MAP kinase signalling cascades. Nature 2001;410:37-40.

-41 Xiao WL, Zhang DZ, Fan CH, Yu BJ. Intermittent Stretching and Osteogenic Differentiation of Bone Marrow Derived Mesenchymal Stem Cells via the p38MAPK-Osterix Signaling Pathway. Cell Physiol Biochem 2015;36:1015-1025.

42 Cantley LC: The Phosphoinositide 3-Kinase Pathway. Science 2002;296:1655-1657.

43 Schiaffino S, Mammucari C: Regulation of skeletal muscle growth by the IGF1-Akt/PKB pathway: insights from genetic models. Skelet Muscle DOI: 10.1186/2044-5040-1-4.

44 Yang H, Shi L, Shi G, Guo Y, Chen D, Chen D, Shi J: Connexin 43 Affects Osteogenic Differentiation of the Posterior Longitudinal Ligament Cells via Regulation of ERK Activity by Stabilizing Runx2 in Ossification. Cell Physiol Biochem 2016;38:237-247.

45 Buitrago CG, Arango NS, Boland RL: Role of VDR in 1 $\alpha$,25-dihydroxyvitamin D3-dependent non-genomic activation of MAPKs, Src and Aktin skeletal muscle cells. J Steroid Biochem Mol Biol 2013;136:125-130.

-46 Yang HS, Li FS, Kong XF, Yuan XX, Wence W, Huang RL, Li TJ, Geng MM, Wu GY, Yin YL: Chemerin regulates proliferation and differentiation of myoblast cells via ERK1/2 and mTOR signaling pathways. Cytokine 2012;60:646-652.

47 Kazuya O, Yosuke N, Eiji W, Peter S.Z, Masataka S, Ryoichi M: Zinc promotes proliferation and activation of myogenic cells via the PI3K/Akt and ERK signaling cascade. Exp Cell Res 2015;333:228-237.

48 Levinger I, Scott D, Nicholson GC, Stuart AL, Duque G, McCorquodale T, Herrmann M, Ebeling PR, Sanders KM: Undercarboxylated osteocalcin, muscle strength and indices of bone health in older women. Bone 2014;64:812.

49 Harslof T, Sikjaer T, Mosekilde L, Langdahl BL, Rejnmark L: Correlations Between Changes in Undercarboxylated Osteocalcin and Muscle Function inHypoparathyroidism. Int J Endocrinol Metab DOI: 10.5812/ijem.38440.

-50 Shea MK, Dawson-Hughes B, Gundberg CM, Booth SL: Reducing Undercarboxylated Osteocalcin With Vitamin K Supplementation Does Not Promote Lean Tissue Loss or Fat Gain Over 3 Years in Older Women and Men: A Randomized Controlled Trial. J Bone Miner Res 2017;32:243-249. 